\title{
Decreased ultrasound echogenity as a thyroid hypofunction marker
}

\author{
D.Dimic, M.Velojic Golubovic, S.Radenkovic, D.Stojic, M Pesic
}

Clinic of endocrinology, Clinical Center Nis, Serbia

\section{Objectives:}

The value of ultrasound in functional disorders can be significant. That is why the question arises on the use of ultrasound examination of thyroid gland and its echogenity as a screening method in early detection of disfunctions, primarily subclinical and clinical forms of hypothyreosis.

\section{Methods:}

\begin{abstract}
Testing included 328 patients. All examinees underwent ultrasound examination of thyroid gland, the blood was taken for determination of FT4, TSH, TPOab andTGab. The patients were divided into two groups. Group A with normal echogenity of thyroid gland tissue, and B with decreased echogenity. Group B was divided into two subgroups, B1 with a mildly decreased and B2 with significantly decreased echogenity.
\end{abstract}

TABLE 1

NUMBER OF PATIENTS IN RELATIONTO ULTRASOUND ECHOGENITYAND TPO ANDTG ANTIBODIES PRESENCE

\begin{tabular}{|c|c|c|c|c|}
\hline \multirow[b]{2}{*}{$\begin{array}{l}\text { Normal echogenity } \\
\text { Group A }\end{array}$} & \multicolumn{2}{|c|}{ No of patients } & TPO increased & TGab positive \\
\hline & 212 & $64.6 \%$ & $18 \quad 8.5 \%$ & $22 \quad 10.4 \%$ \\
\hline $\begin{array}{l}\text { Decreased } \\
\text { echogenity } \\
\text { Group B }\end{array}$ & 116 & $35.4 \%$ & $56 \quad 48.3 \% *$ & $48 \quad 41.4 \% *$ \\
\hline $\begin{array}{l}\text { Mildly decreased } \\
\text { echogenity } \\
\text { Group B1 }\end{array}$ & 91 & $27.7 \%$ & $34 \quad 37.3 \%^{*}$ & $30.7 \% *$ \\
\hline $\begin{array}{l}\text { Significantly } \\
\text { decreased } \\
\text { echogenity }\end{array}$ & 25 & $7.7 \%$ & $2288.0 \% * ¥$ & $2080.0 \% * ¥$ \\
\hline
\end{tabular}

Group B2
TABLE 2

MEAN VALUES OF TBOab, Ft4 ANDTSH IN RELATIONTO ULTRASOUND ECHOGENITY

\begin{tabular}{|c|c|c|c|}
\hline & TPOab U & fT4 $\mathrm{nmol} / \mathrm{l}$ & TSH $\mathrm{mlU} / \mathrm{l}$ \\
\hline $\begin{array}{l}\text { Normal echogenity } \\
\text { Group A }\end{array}$ & $58.5 \pm 18.4$ & $14.6 \pm 5.5$ & $1.05 \pm 0.34$ \\
\hline $\begin{array}{l}\text { Decreased } \\
\text { echogenity } \\
\text { Group B }\end{array}$ & $339.3 \pm 57.8^{*}$ & $10.1 \pm 2.9$ & $5.04 \pm 1.98$ \\
\hline $\begin{array}{l}\text { Mildly decreased } \\
\text { echogenity } \\
\text { Group B1 }\end{array}$ & $248.0 \pm 45.9 *$ & $11.2 \pm 3.4^{*}$ & $3.82 \pm 2.12^{*}$ \\
\hline $\begin{array}{l}\text { Significantly } \\
\text { decreased } \\
\text { echogenity } \\
\text { Group B2 }\end{array}$ & $670 \pm 116.7^{*} ¥$ & $8.8 \pm 3.8 * ¥$ & $8.75 \pm 3.36 * ¥$ \\
\hline
\end{tabular}

TABLE 3

NUMBER OF PATIENTS ACCORDING TO THEVALUES OF TSH AND ULTRASOUNDECHOGENITY

\begin{tabular}{|c|c|c|c|}
\hline & $\mathrm{TSH}<4 \mathrm{mIU} / \mathrm{I}$ & TSH $4-10 \mathrm{mlU} / \mathrm{l}$ & $\mathrm{TSH}>10 \mathrm{mlU} /$ \\
\hline $\begin{array}{l}\text { Normal echogenity } \\
\text { Group A }\end{array}$ & $21099 \%$ & $1 \%$ & 0 \\
\hline $\begin{array}{l}\text { Decreased } \\
\text { echogenity } \\
\text { Group B }\end{array}$ & $87 \quad 75 \% *$ & $2118.1 \%^{*}$ & $6.9 \% *$ \\
\hline $\begin{array}{l}\text { Mildly decreased } \\
\text { echogenity } \\
\text { Group B1 }\end{array}$ & $8189 \% *$ & $8.8 \% *$ & $2.2 \% *$ \\
\hline $\begin{array}{l}\text { Significantly } \\
\text { decreased } \\
\text { echogenity }\end{array}$ & $24.0 \% * ¥$ & $1352.0 \% * ¥$ & $6 \quad 24 \% * ¥$ \\
\hline
\end{tabular}

TPO antibodies, TSH and TG antibodies positivity and their mean values in group B are significantly higher, as well as in subgroups $B 1$ and $B 2$, in relation to group $A, p<0.001$. In group $A$ only two examinees $(1 \%)$ were indicated with subclinical hypothyreosis. In group $B$ the sublinical hypothyreosis was indicated in 21 , while the clinical hypothyreosis was indicated in 8 examinees. $29(25 \%)$ suffered of thyroid gland altered function. In subgroup B1 8 examinees were indicated with subclinical and 2 with clinical hypothyreosis. 10 examinees (11\%) suffered of altered thyroid function. In group B2 the subclinical was found in 13 examinees, while the clinical hypothyreosis was found in 6.19 examinees $(76 \%)$ suffered of altered thyroid function. sublinical and clinical hypothyreosis. Decreased ultrasound echogenity represents the significant marker of altered thyroid gland function. In these persons we have determined the high percentage of subclinical and clinical hypothyreosis frequency. 